\title{
FACTORES CRÍTICOS QUE IMPACTAN LAS FINANZAS PÚBLICAS DE LOS GOBIERNOS LOCALES DE LA REGIÓN TACNA
}

\section{CRITICAL FACTORS THAT IMPACT THE LOCAL GOVERNMENTS' PUBLIC FINANCES OF THE TACNA REGION}

\author{
Percy Vilchez Olivares \\ Universidad Nacional Mayor de San Marcos \\ Doctor en Ciencias Contables Empresariales de la Universidad Nacional Mayor de San Marcos de Lima. \\ Correo: pvilchezo@unmsm.edu.pe (Autor Corresponsal) \\ Roberto Neyra Urquiza \\ Universidad Nacional Jorge Basadre Grohmann \\ Magister en Contabilidad con mención en Auditoría de la Universidad Nacional Jorge Basadre Grohmann de Tacna. \\ Tania Nolberto Acosta \\ Universidad de Huánuco \\ Magister en Contabilidad con mención en Auditoría y Tributación de la Universidad de Huánuco.
}

[Recibido: 08/11/2017 Aceptado: 03/04/2018]

\begin{abstract}
RESUMEN
El objetivo de la investigación es presentar las causas y efectos que se generan en las finanzas públicas de los gobiernos locales de la región de Tacna, a través de las variables de ingresos, gastos, inversión, financiación, entre otras. Una gestión económica y administrativa eficiente de las finanzas públicas en las municipalidades implica generar recursos propios suficientes, permite tener resultados positivos en el manejo de los fondos y esta a su vez permite una mejor calidad de servicio que se brinda a la población. El diseño investigativo utilizado es de tipo deductivo y sistémico, para dicho efecto se entrevistaron a los alcaldes, gerentes municipales, contadores, encargados de las áreas administrativas y financieras de las municipalidades; con el objetivo de determinar los factores que influyen la gestión de las finanzaspúblicas. La población objetiva fueron 4 municipalidades provinciales y 23 municipalidades distritales que forman parte de la región Tacna. Los resultados obtenidos nos permite señalar que en el año 2014 un 25,93\% de las municipalidades de la región Tacna dependen del 90\% de los ingresos por transferencias del gobierno central, es decir un $10 \%$ de los fondos provienen de ingresos propios, en el tema presupuestal el $29,63 \%$ de las municipalidades. Respecto al presupuesto el $29,63 \%$ de las municipalidades obtuvo déficit o superávit presupuestario mayor al $10 \%$, un $40.74 \%$ obtuvo un equilibrio en la liquidación efectiva del presupuesto, el $33,33 \%$ de las municipalidades han destinado menos del $65 \%$ del ingreso total en inversión, también se observa que el 14,81\% cuenta con información financiera con atraso mayor a 6 meses y el $44.44 \%$ de las municipalidades no tienenestudios de costos elaborados y/o actualizados.
\end{abstract}

Palabras clave : Finanzas públicas, gobiernos locales, infraestructura, planificación.

\begin{abstract}
The objective of the investigation is to present the causes and effects that are generated in the finances of the countries of the Tacna region, through the variables of income, expenses, investment, financing, among others. An economic and efficient management of public finances in the municipalities implies generating sufficient resources, allows for positive results in the management of funds and, in turn, allows a better quality of service that can be provided. The investigative design is deductive and systemic, for this purpose it was given to the mayors, municipal managers, accountants, in charge of the administrative and financial areas of the municipalities; with the objective of determining the factors that influence the management of public finances. The objective population was 04 provincial municipalities and 23 district municipalities that are part of the Tacna region. The results obtained indicate that in $2014,25.93 \%$ of the municipalities in the Tacna region depend on $90 \%$ of the revenues of the central government, that is, $10 \%$ of the funds obtained from their own income, in the subject budget $29.63 \%$ of the municipalities. Regarding the budget, $29.63 \%$ of the municipalities obtained a budget deficit or surplus greater than $10 \%, 40.74 \%$ obtained a balance in the effective liquidation of the budget, $33.33 \%$ of the municipalities had a cost lower than $65 \%$ of the total income in investment, it is also observed that $14.81 \%$ have financial information with arrears greater than 6 months and $44.44 \%$ of municipalities do not have an elaborated and / or updated cost budget.
\end{abstract}

Keywords: Public finances, local governments, infrastructure, planning.

( ) Los autores. Este artículo es publicado por la Revista Quipukamayoc, Universidad Nacional Mayor de San Marcos. Este es un artículo de acceso abierto, distribuido bajo los términos de la Licencia Creative Commons Atribución-NoComercial-Compartirlgual 4.0 Internacional.(http://creativecommons.org/licenses/by-nc-sa/4.0/), que permite el uso no comercial, distribución y reproducción en cualquier medio, siempre que la obra original sea debidamente citadas. 


\section{INTRODUCCIÓN}

Los antecedentes iniciales de las finanzas públicas en la sociedad surgen desde 3,000 a.C. donde la sociedad China ya tenía tesoreros reales y personas para llevar las cuentas de los tributos. Asimismo, en Egipto y lo refiere la sagrada biblia, José era el encargado de administrar el recurso del imperio para el sostenimiento de todos, a esto contribuyeron los escolásticos siglo XVI, los mercantilistas siglo XVII, los cameralistas, fisiócratas, los clásicos entre otros.

EL desarrollo del mundo contemporáneo y la creación de nuevas institucionales locales y regionales para administrar los servicios comunes de la sociedad permitieron el surgimiento de los gobiernos locales y municipales. Estas entidades y muy específicamente en nuestro país generalmente no aplican conceptos y técnicas modernas de gestión municipal; situación diferente que se presenta en países como Estados Unidos y Reino Unido que si aplican éxitos modelos de gestión municipal.

En los últimos años se ha generado en diversas entidades municipales de tipo regional o local un interés por revisar los modelos de gestión municipal y conocer cuáles son los factores que influyen o afectan la consecución de una gestión municipal eficiente. $\mathrm{Al}$ respecto, en un estudio sobre gestión del desarrollo local en los municipios realizado por Machado et al. (2007, p.1) explicaron "La globalización y la revolución de la informática y las telecomunicaciones, entre otras, han provocado incertidumbre y complejidad para analizar los fenómenos y procesos que inciden en el desarrollo. Es por ello que, durante los últimos años del siglo XX, comenzó a gestarse una tendencia hacia el análisis de lo local, perspectiva que surge como consecuencia de la inviabilidad de las diferentes estrategias de desarrollo efectuadas y como alternativa para lo- grar una mayor efectividad y la sostenibilidad en las transformaciones que se implementan".

Debido a lo anterior, diversas entidades vienen desarrollando diversas actividades y experiencias exitosas en las que incorporan nuevos modelos de gestión municipal y local utilizando tecnología de la información en los procesos de gestión de las entidades. De esta forma buscan ser eficientes en la administración de los ingresos y gastos, con la finalidad de reducir el gasto público y lograr una estabilidad económica.

A pesar de lo anterior, existen un gran grupo de entidades que no logran tener un manejo eficiente de sus recursos y consecuentemente tiene un inadecuado manejo del presupuesto asignado generando un desequilibrio económico de estas entidades, incluso en muchas entidades los gastos públicos superan sus ingresos, aumentando así significativamente su endeudamiento público.

Cabe señalar al economista investigador del ICEFI, Juan José Urbina (2016) advierte que "El Salvador se enfrenta actualmente a dos situaciones. Una de ellas es la cercanía de una crisis de iliquidez, por la falta de recursos inmediatos que le permitan cumplir con todas sus obligaciones. Esta situación, señaló, puede provocar un default. Pero otra situación que complica el escenario financiero del Gobierno es la incapacidad de lograr acuerdos mínimos que permitan solucionar este problema de recursos. El estudio indica que El Salvador es uno de los países con mayor riesgo de insostenibilidad fiscal. ¿Qué debemos entender por insostenibilidad fiscal? ¿Se trata de un default?. El término sostenibilidad es la capacidad de los gobiernos para mantener constante su nivel de endeudamiento respecto al PIB, respecto a los ingresos fiscales, entre otros. Una vez los gobiernos pierden dicha capacidad, y su nivel de endeudamiento crece constantemen- te y a una razón mayor que el crecimiento económico y el crecimiento de los ingresos fiscales, nos encontramos ante una situación de insostenibilidad, la cual puede derivar en una crisis fiscal generada por endeudamiento. Los default se dan cuando el tamaño del endeudamiento presiona las finanzas públicas hasta un nivel en que los recursos disponibles no son suficientes para honrar los pagos relacionados al endeudamiento..."

Por lo planteado por el investigador Urbina, es claro que el Hermano país de El Salvador se enfrenta a una crisis de iliquidez por falta de recursos que le permitan atender las obligaciones más urgentes, consecuentemente se puede presentar un default en el momento que se deje de cumplir con sus obligaciones, pero según opinión de Urbina et al. (2016) existe "Una falta de capacidad y claridad para lograr acuerdos políticos inmediatos en materia fiscal para solventar el problema de liquidez". En ese sentido, existe un riesgo de sostenibilidad financiera de ámbito fiscal en El Salvador y por lo tanto es necesario una reforma profunda en los ingresos y gasto público, en forma inmediata, debido a que un atraso en atender las necesidades puede generar conflictos sociales teniendo como actores a la población y a las entidades con resultados impredecibles.

Al respecto, Archundia (2009) precisa en su tesis doctoral titulada "El impacto tributario de la economía informal en México en busca de una propuesta estructural" precisa que los problemas administrativos que se presentan en los municipios en general y que desde luego impactan negativamente en la recaudación, se muestran por la calidad insuficiente de la administración pública porque evita que los ciudadanos tengan credibilidad en el Gobierno, además que inhiben evitar que los ciudadanos identifiquen las consecuencias positivas y negativas de contribuir. 
Hoy en día, a nivel nacional las Municipalidades para el logro de sus fines y objetivos, así como para el desempeño de sus funciones y actividades, afrontan diversos problemas en forma cotidiana, los que se traducen en severas limitaciones en el campo organizacional, administrativo, económico, financiero, tecnológico, entre otros, que no permiten satisfacer adecuadamente las necesidades de la población en el ámbito de su competencia. En la provincia Constitucional del Callao, Huarcaya (2011), afirma en su investigación, que "La Municipalidad ha empleado un rol que se traduce en las siguientes acciones: una política de descentralizar las funciones, las responsabilidades y la toma de decisiones; dotando de más libertad a los colaboradores para que puedan ejercer sus funciones, todo esto enmarcado dentro del ámbito de sus atribuciones, la cual está contemplada en el MOF, logrando así una adecuada gestión administrativa eficaz de los recursos públicos y de atención al ciudadano”.

De manera complementaria Mendoza (2016) señala "Hay que tener en cuenta, que la evolución de las finanzas públicas se ha acondicionado a la economía internacional, de tal forma que en el año 2011 creció en 16\% los ingresos, en 2016 se estima una baja más o menos de $6 \%$ porque hay razones internacionales para que esto suceda y el gasto público tiene un crecimiento de $5 \%$ al año lo cual genera una brecha que se manifiesta en el déficit fiscal".

Por lo expuesto, es necesario que se planteen nuevos modelos de gestión y que se revisen los factores que afectan la calidad de la gestión en el ámbito económico y administrativo, pues de esto depende poder lograr un manejo eficiente de los recursos, presupuestos asignados y consecuentemente contar con una capacidad para un adecuado y eficiente manejo de las inversiones y gastos públicos en las municipalidades.
Se aplicó el tipo de investigación descriptiva; se hizo uso de los métodos investigativo científico, deductivo y sistémico; es importante mencionar que en la población objetiva fueron consideradas las veintitrés (23) municipalidades distritales y (4) provinciales de la región Tacna. En la presente investigación se utilizó la técnica de la encuesta, se aplicó un cuestionario de Autoevaluación de la Gestión Municipal, bajo la dimensión de Desarrollo Organizacional, creada por el Grupo Gestor conformado por los organismos GTZ-cooperación técnica alemana, Research Triangle Institute (RTI/USAID), Programa de Transparencia y Gobernabilidad (TAG/ USAID), Programa de las Naciones Unidas para el Desarrollo (PNUD), entre otras; mientras que para observar los factores críticos, se obtuvieron del Formulario No 01 del Registro Nacional de Municipalidades (RENAMU) amparada en la Ley No 27563 del 25 de noviembre del 2001, a cargo del Instituto Nacional de Estadística e Informática (INEI) correspondiente al año 2015. Al respecto, el RENAMU, dispone de información estadística de las Municipalidades Provinciales, Distritales y de Centros Poblados, con el propósito de generar indicadores municipales que sirvan de apoyo a la gestión regional y local, para una adecuada planificación y toma de decisiones.

Diseño

Con respecto al diseño del RENAMU, se han considerado las secciones de datos generales de la municipalidad, infraestructura, recursos humanos y planificación municipal. Mientras que para el instrumento de autoevaluación municipal se consideraron los indicadores de operar bajo objetivos y metas; ordenamiento del territorio; formación del personal; sistemas mecanizados.

El Formulario del RENAMU y la encuesta de Autoevaluación de Desarrollo Organizacional Municipal, se distribuyó en versión impresa a todas las municipalidades provinciales y distritales del país y también, se obtuvo el formulario a través de la página web del INEI http://www. inei.gob.pe; el Alcalde o funcionario de la municipalidad es el responsable de los datos consignados en el formulario, en cumplimiento de la Ley $\mathrm{N}^{\circ}$ 27563 y del D.S. $N^{\circ}$ 033-2002-PCM que reglamenta la Ley, las preguntas del Formulario 01 fueron referidas al periodo del 01 de enero al 31 de diciembre de 2015. De la misma manera se suministró el formulario de Autoevaluación del Desarrollo Organizacional Municipal.

Los resutados se exponen de la siguiente manera: Los resultados referentes a la caracterización de las municipalidades, para luego analizar los resultados concernientes propiamente a la investigación.

\section{Finanzas municipales}

En función a la buena administración financiera de los recursos públicos se han adoptado mejoras a las tecnologías, en la gestión y planificación en las finanzas públicas. En la tabla 1 se muestran los resultados del nivel de logro en las finanzas municipales de la gestión municipal de la región Tacna en el año 2014, entre las cuales 1 de ellas calificó con un nivel "bajo", esta fue la municipalidad distrital de Curibaya de la provincia de Candarave, 15 alcanzaron una calificación de nivel "medio" y finalmente 11 municipalidades alcanzaron un "alto" nivel de calificación entre ellas, las municipalidades de Tacna, Alto de la Alianza, Calana, Inclán, Palca, Pocollay y Coronel Gregorio Albarracín de la provincia de Tacna, la municipalidad de Candarave, Jorge Basadre e Ilabaya, finalmente la municipalidad de Estique-Pampa de la provincia de Tarata. 
Tabla 1. Nivel de logro en Finanzas Municipales de la Gestión Municipal, según municipalidades provinciales y distritales de la región Tacna, 2014.

Fuente: Elaboración propia, basada en la encuesta de gestión municipal en el departamento de Tacna, 2014.

\begin{tabular}{|c|c|}
\hline Municipalidad provincial y distrital & $\begin{array}{c}\text { Nivel de logro en finanzas } \\
\text { municipalidades }\end{array}$ \\
\hline Tacna & Alto \\
\hline Alta de la Alianza & Alto \\
\hline Calana & Alto \\
\hline Ciudad Nueva & Medio \\
\hline Inclán & Alto \\
\hline Pachía & Medio \\
\hline Palca & Alto \\
\hline Pocollay & Alto \\
\hline Sama & Medio \\
\hline Coronel Gregorio Albarracín Lanchipa & Alto \\
\hline Candarave & Alto \\
\hline Cairani & Medio \\
\hline Camilaca & Medio \\
\hline Curibaya & Bajo \\
\hline Huanuara & Medio \\
\hline Quilahuani & Medio \\
\hline Jorge Basadre & Alto \\
\hline Ilabaya & Alto \\
\hline Ite & Medio \\
\hline Tarata & Medio \\
\hline Héroes Albarracín & Medio \\
\hline Estique & Medio \\
\hline Estique-Pampa & Alto \\
\hline Sitajara & Medio \\
\hline Susapaya & Medio \\
\hline Tarucachi & Medio \\
\hline Tiraco & Medio \\
\hline
\end{tabular}

Capacidad de potenciar y optimizar la recaudación municipal

La figura 1 muestran los resultados de las municipalidades según porcentaje de ingresos propios relacionados con los ingresos totales en el año 2014, en el $25,93 \%$ de las municipalidades los ingresos propios representan menos del $10 \%$ de sus ingresos totales, en el $55,56 \%$ los ingresos propios van entre $10 \%$ y $25 \%$ y finalmente en el $18,52 \%$ de las municipalidades de la región, sus ingresos propios representan más del $25 \%$ de sus ingresos totales. 


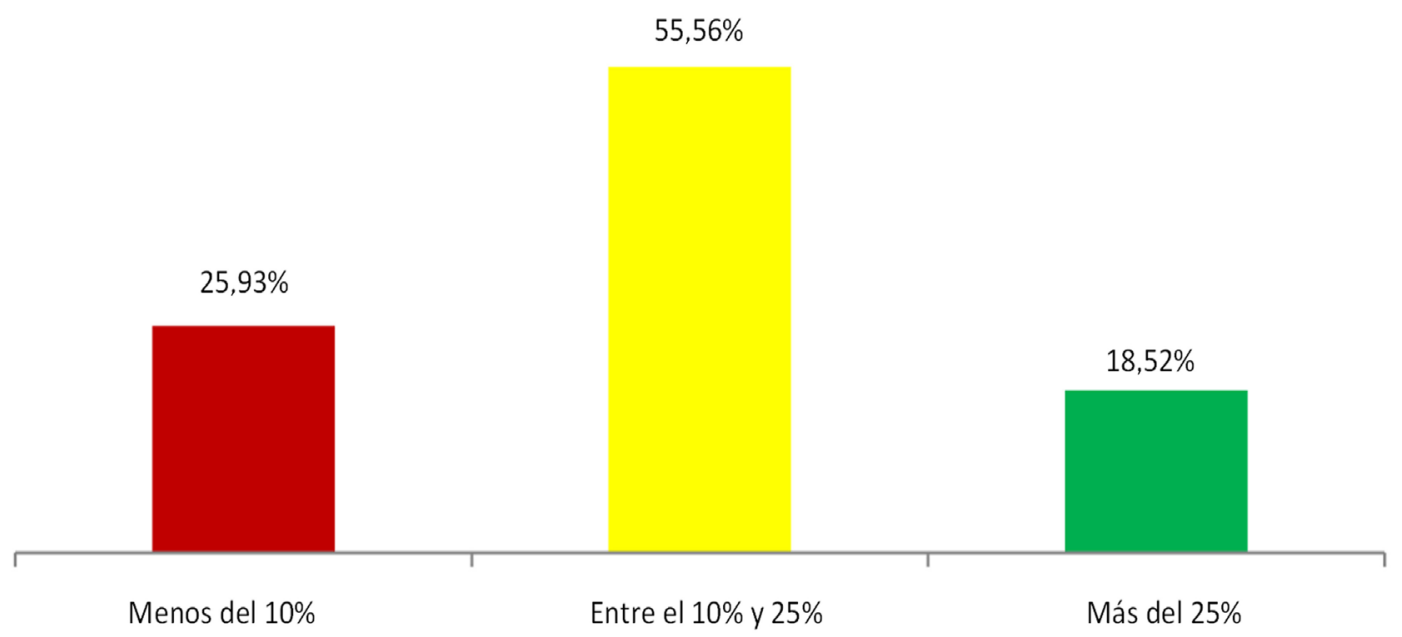

Figura 1. Municipalidad que cuenta con un Plan Operativo Anual (POA) que se usa para monitorear y evaluar las actividades, según condición, 2015.

Fuente: Elaboración propia, basada en la encuesta de gestión municipal en el departamento de Tacna, 2014.

La figura 2 muestra los resultados de las municipalidades según porcentaje de la mora tributaria que ha sido re- cuperada con respecto a la mora total en el año 2014, el 59,26\% de las municipalidades recuperaron hasta el 5\% de la mora, el 29,63\% recuperó del 5\% hasta el $15 \%$ y finalmente el 11,11\% recuperaron más del 15\%.

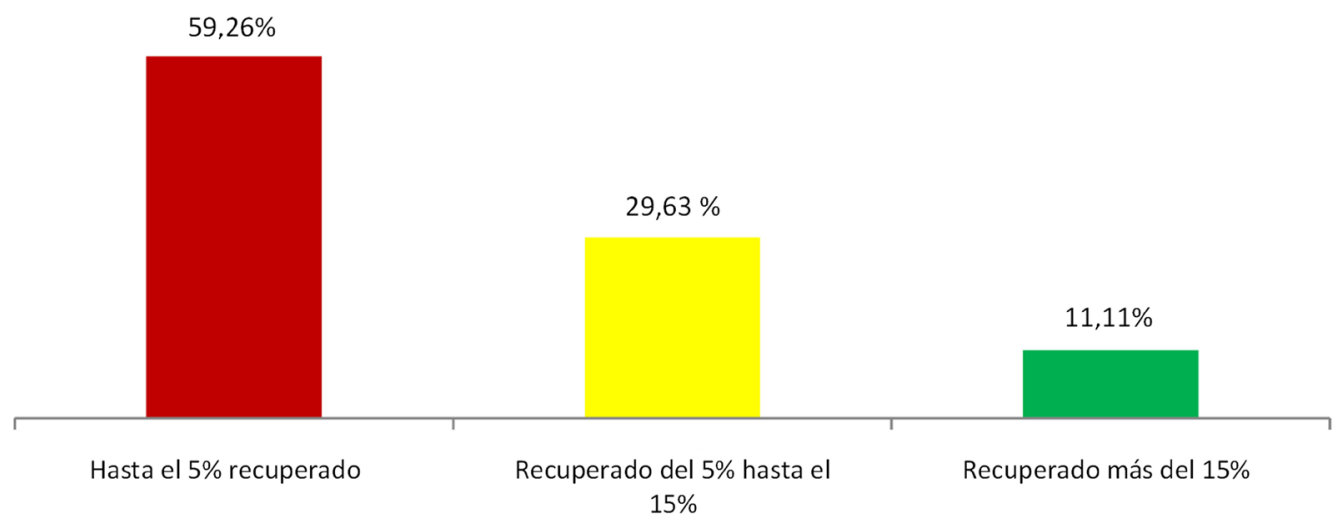

Figura 2. Municipalidades según porcentaje de la mora tributaria que ha sido recuperada con respecto a la mora total, 2014.

Fuente: Elaboración propia, basada en la encuesta de gestión municipal en el departamento de Tacna, 2014. 
Capacidad de manejo presupuestario

La figura 3 muestra los resultados de las municipalidades según liquidación efectiva del presupuesto total proyectado. En el año 2014, el 29,63\% de las municipalidades obtuvo déficit o superávit presupuestario mayor al $10 \%$, un $29,63 \%$ obtuvo un déficit o superávit presupuestario menor al
$10 \%$ y el $40,74 \%$ de las municipalidades obtuvo un equilibrio en la liquidación efectiva del presupuesto total proyectado.

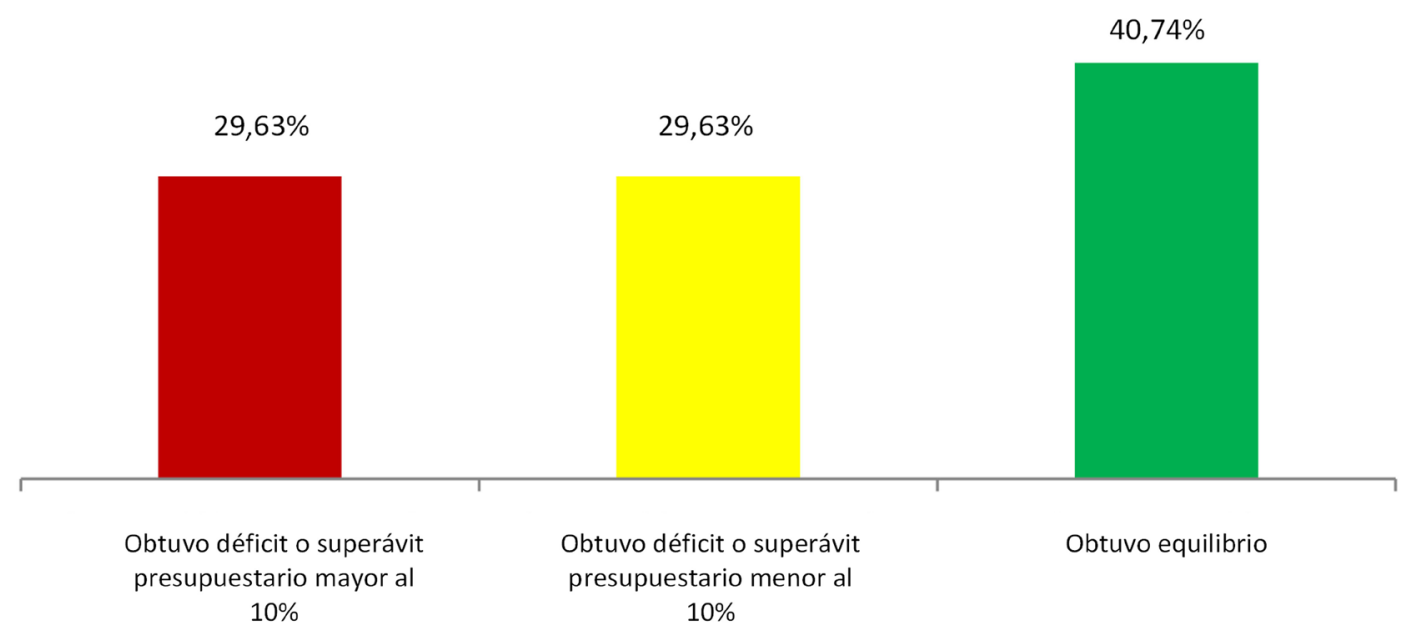

Figura 3. Municipalidades según liquidación efectiva del presupuesto total proyectado, 2014.

Fuente: Elaboración propia, basada en la encuesta de gestión municipal en el departamento de Tacna, 2014.

La figura 4 muestra los resultados de las municipalidades según eficiencia en la ejecución de la inversión en el año 2014 , el $33,33 \%$ de las munici- palidades han destinado menos del $65 \%$ del ingreso total en inversión, el $37,04 \%$ destinó entre el $65 \%$ y $75 \%$ del ingreso total en inversión y finalmen- te el $29,63 \%$ de las municipalidades destinó más del 75\% del ingreso total en inversión.

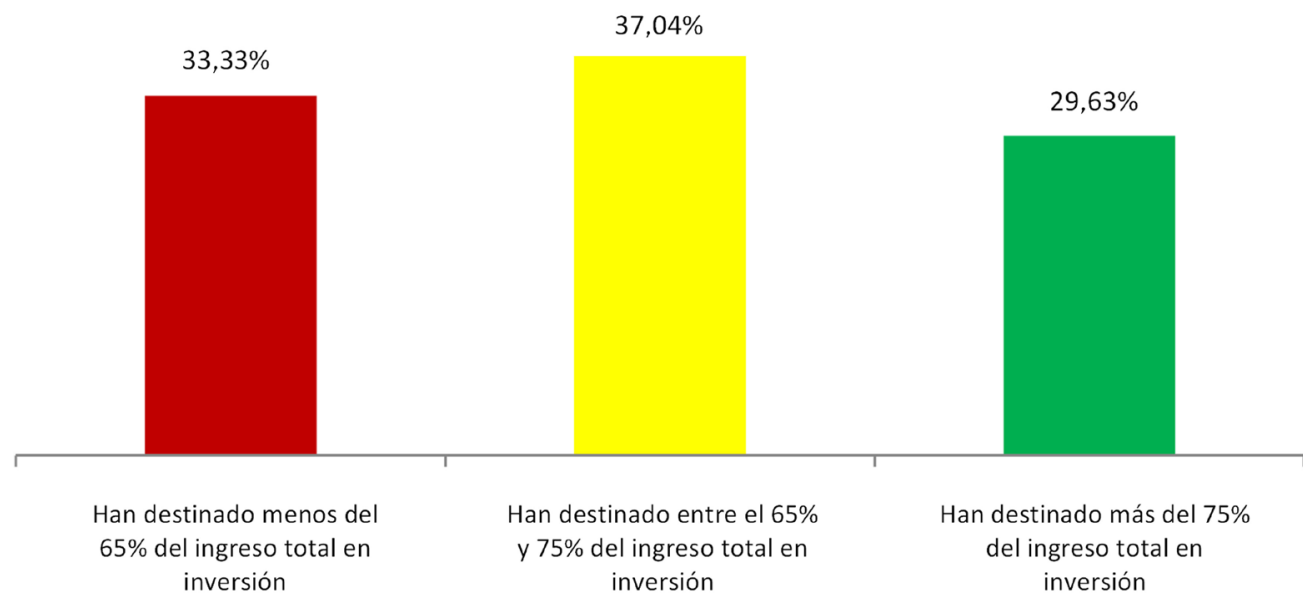

Figura 4. Municipalidades según eficiencia en la ejecución de la inversión, 2014.

Fuente: Elaboración propia, basada en la encuesta de gestión municipal en el departamento de Tacna, 2014. 
Eficiencia en la ejecución de la inversión

La figura 5 muestra los resultados de las municipalidades según relación de la deuda bancaria amortizada con respecto al ingreso total de la municipalidad en el año 2014, se observa que $22,22 \%$ de las municipalidades registra en el indicador una relación "Ma- yor que 1", en el 3,70\% de municipios la relación es "Igual a 1" y finalmente el 74,07\% dio como resultado en el indicador una relación "Menor que 1 ".

\section{$74.07 \%$}

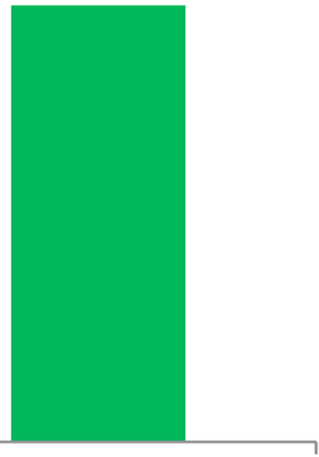

\section{Cuando la relación es} menor que 1

Figura 5. Municipalidades según relación de la deuda bancaria amortizada con respecto al ingreso total de la municipalidad, 2014.

Fuente: Elaboración propia, basada en la encuesta de gestión municipal en el departamento de Tacna, 2014.

\section{Capacidad administrativa financiera}

La figura 6 muestra los resultados de las municipalidades según tenencia de información financiera oportuna y confiable. En el año 2014, se observa que $14,81 \%$ de las municipalidades cuentan con a información financiera, pero con un "Atraso mayor a 6 meses", el $25,93 \%$ dispone de esta con un atraso entre 1 y 6 meses y finalmente el $59,26 \%$ de las municipalidades de la región, cuenta con información financiera con un atraso menor a 1 mes.

$59,26 \%$

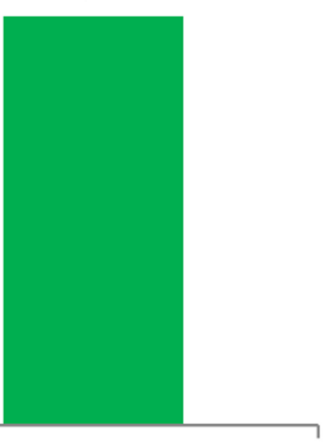

Presenta un atraso mayor a Presenta un atraso entre 1 y 6 meses
Presentando un atraso menor a 1 mes

Figura 6. Municipalidades según tenencia de información financiera oportuna y confiable, 2014. Fuente: Elaboración propia, basada en la encuesta de gestión municipal en el departamento de Tacna, 2014. 
La figura 7 muestra los resultados de las municipalidades según tenencia de estudios de costos elaborados y/o actualizados para el cálculo de las tasas por los servicios municipales pres- tados En el año 2014, se observa que el $44,4 \%$ de las municipalidades "No poseen estudios de costos", el 25,9\% "Poseen el estudio de costos pero no se utilizan para el ajuste de tasas" y finalmente el $29,6 \%$ de las municipalidades de la región "Utilizan el estudio de costos para el cálculo de las tasas".

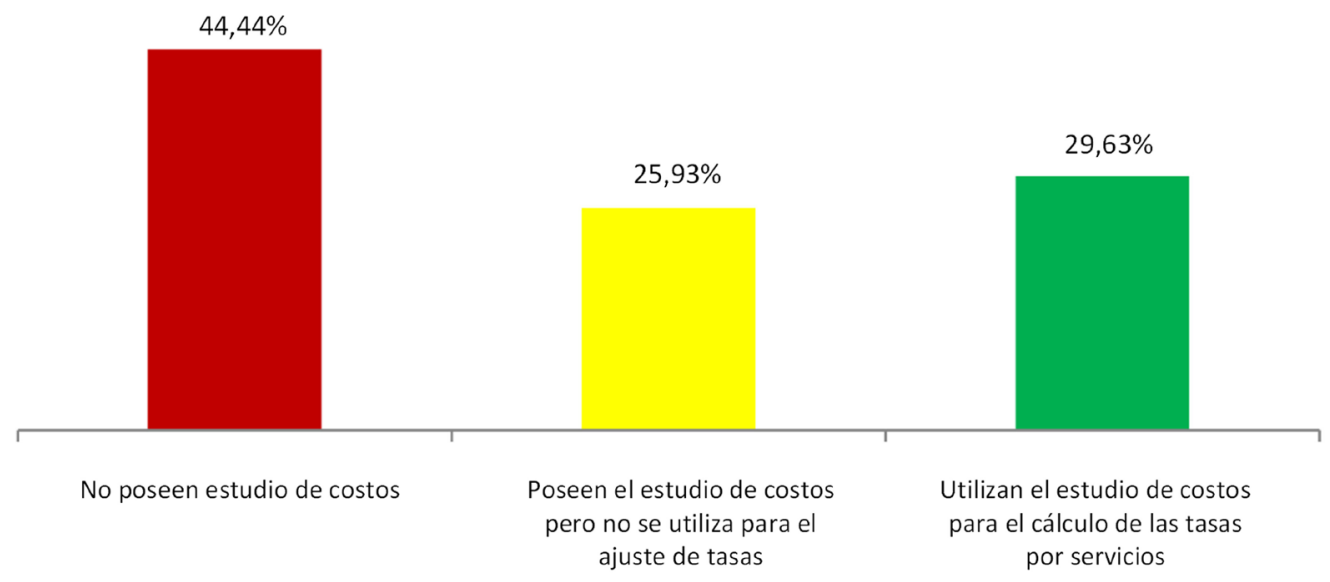

Figura 7. Municipalidades según tenencia de estudios de costos elaborados y/o actualizados para el cálculo de las tasas por los servicios municipales prestados, 2014.

Fuente: Elaboración propia, basada en la encuesta de gestión municipal en el departamento de Tacna, 2014.

La figura 8 muestra los resultados de las municipalidades según porcentaje del presupuesto ejecutado invertido en proyectos dirigidos a sectores con vulnerabilidad, en el año 2014, se observa que $22,22 \%$ de las municipalidades ejecutó "Hasta el 10\% de la inversión”, la mayoría de municipalidades
$48.15 \%$ ejecutó entre el $11 \%$ y $24 \%$ de inversión y finalmente el 29,63\% alcanzó "Más del 25\% de la inversión".

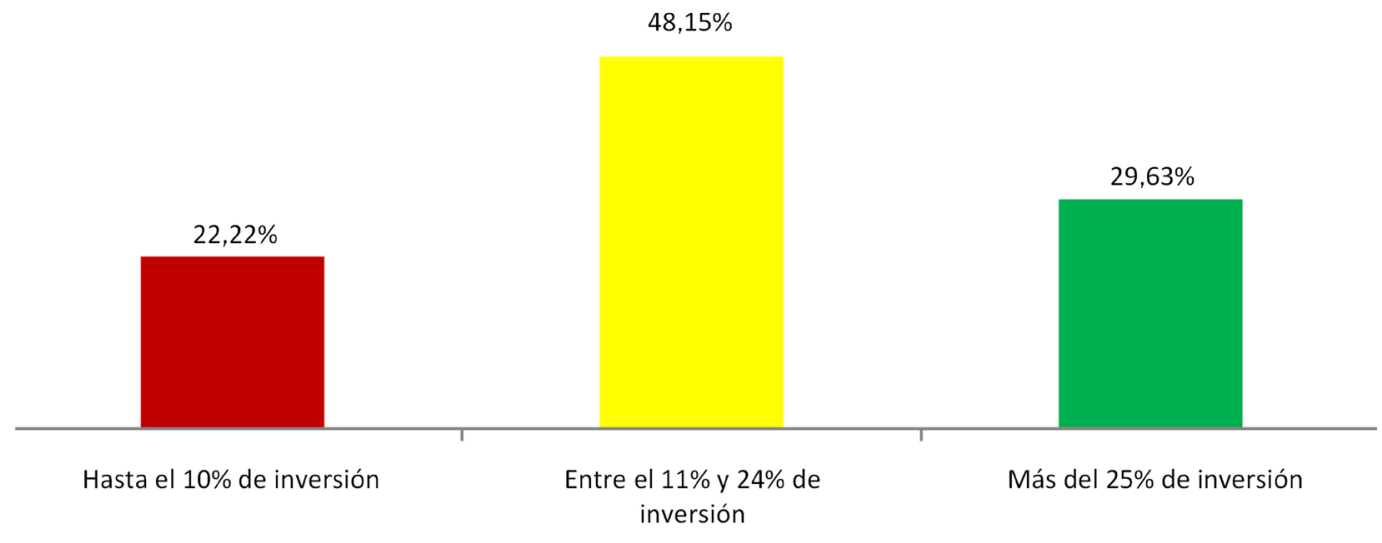

Figura 8. Municipalidades según porcentaje del presupuesto ejecutado invertido en proyectos dirigidos a sectores con vulnerabilidad social, 2014

Fuente: Elaboración propia, basada en la encuesta de gestión municipal en el departamento de Tacna, 2014. 


\section{CONCLUSIONES}

Del $100 \%$ de los ingresos de las municipalidades de la región Tacna, un $90 \%$ corresponden a los ingresos por transferencias del Gobierno Central y menos del $100 \%$ corresponden a fondos que provienen de ingresos propios; esto se debe al poco ingreso que genera en forma directa por las municipalidades, y esto es consecuencia del bajo nivel de servicios municipales que se brinda a los contribuyentes.

Existe un bajo nivel de recuperos de las moras tributarias en las municipalidades, por lo que es necesario, revisar el sistema de fijación de moras y recaudación. Los resultados nos indican que el $11 \%$ constituyen recuperaciones mayores al 15\%. De mantenerse esta situación no se generaran recursos suficientes para atender los gastos de gestión.

Del $100 \%$ de municipalidades, los resultados de déficit o superávit presupuestario mayor o menor al $10 \%$ es del $60 \%$ y sólo un $40 \%$ obtuvo equilibrio presupuestal. Esta situación afecta el programa de ejecución de inversiones y gastos corrientes en las municipalidades.

En el año 2014 se ha presentado un bajo nivel de inversiones en las municipalidades sólo un 33\% destino menos del $65 \%$ del ingreso total en inversiones, lo cual afecta los programas de modernización y remodelación de la infraestructura pública.

Del $100 \%$ de las municipalidades el $22 \%$ de las entidades presenta un indicador de deuda bancaria amortizada con respecto al ingreso total mayor que 1 , lo cual significa que a nivel del sistema existe una alta presencia de endeudamiento bancario.
De 27 municipalidades el 59\% presenta información financiera con un atraso menor a 1 mes, el cual es considerado un tiempo razonable. Pero existe un $41 \%$ de entidades que presenta con atraso mayor a 1 mes la información financiera (el 15\% presenta un atraso mayor a 6 meses), lo cual afecta el proceso de toma de decisiones y el control de gestión. Es necesario que los órganos de gobierno de las municipalidades soliciten a las áreas pertinentes la entrega de información oportuna.

Del $100 \%$ de las entidades analizadas se observa que un $44 \%$ no presenta estudios de costos, lo cual afecta los procesos de gestión municipal respecto a la calidad en la toma de decisiones. Asimismo, no contar con estudios de costos no permite actualizar las tasas y contribuciones que se establecen para una adecuada cobertura de los servicios prestados.

De las 27 municipalidades de la región Tacna, sólo el 30\% de las municipalidades ejecutó el más del 25\% de la inversión; es decir el $70 \%$ ejecuto niveles menores del $25 \%$ en programas de inversión. Estos resultados afectan la ejecución de los programas de inversiones en cumplimiento y en aplazamiento de la ejecución; este bajo nivel de inversión justificado por el bajo nivel de recaudación genera un déficit mayor en la cobertura de necesidades sociales de la población, acrecentando los niveles de pobreza y un aumento de la insatisfacción de la población respecto al logro de un bienestar social.

\section{REFERENCIAS BIBLIOGRÁFICAS}

Archundia, E. (Noviembre de 2009). El Impacto Tributario de la Economía Informal en México en busca de una Propuesta Estructural. México, D.F.

Asencio, P. (2006). El libro de la gestión municipal. España: Ediciones Díaz de Santos.

Castillo, J. (2004). Reingeniería y gestión municipal. Lima : Universidad Nacional Mayor de San Marcos.

Flores, W., Borrego, I., \& Salvo, M. (2010). La gestión de programas de inversión pública en el Perú: Propuesta metodológica para mejorar el impacto en iniciativas de desarrollo regional. XIV - AEIPRO, EEAGrants ,Madrid

Huarcaya, M. (2011). Aplicación del empowerment para lograr una gestión eficiente en la municipalidad provincial del Callao. Provincia Constitucional del Callao: Universidad Nacional del Callao.

INEI. (15 de Octubre de 2014). Ficha Técnica: Registro Nacional de $\mathrm{Mu}$ nicipalidades. Registro Nacional de Municipalidades - RENAMU 2013. Lima, Perú: INEI.

INEI, (2010). Estadística de la Gestión Municipal e Infraestructura. Lima: INEI.

Ministerio de Economía y Finanzas. (27 de Abril de 2013). Ejecución de Proyectos del MEF. Obtenido de: http://ofi.mef.gob.pe/bingos/transparencia_pi/mensual/

Machado, H. (2007). Gestión del desarrollo local en los municipios: la iniciativa municipal como experiencia de cambio en la provincia 
de Matanzas. Pastos y Forrajes [online]., 30,(5), 1-1.

Mendoza, W. (04 de 09 de 2016). Expectativa por Kuczynski haría que inversión privada suba en $5 \%$ el 2017. Recuperado el 02 de 10 de 2016, de La Republica.pe
Ramos, H. (2012). Propuesta de un modelo de gestión para mejorar la dirección municipal de La provincia de Otuzco. Industrial Data, $\mathrm{XV}(1), 35-44$.

Soria, B. (2007). Diccionario Municipal Peruano. Herramientas para la
Gestión Municipal. Lima : INICAM - Instituto de Investigación y Capacitación Municipal.

Urbina, J. (08 de 09 de 2016). La crisis de iliquidez puede generar default. Recuperado el 02 de 10 de 2016, de El mundo: http://elmundo.sv/ la-crisis-de-iliquidez-puede-generar-default/ 